\title{
Editorial: Security, Search and Rescue Robotics
}

\author{
Richard Voyles • Julie A. Adams
}

Published online: 8 July 2011

(C) Springer Science+Business Media B.V. 2011

This special issue collects some of the best papers from the 2009 IEEE International Workshop on Safety, Security and Rescue Robotics held at the University of Denver in late 2009. This single-track workshop, in its seventh year, has become the premier venue for search and rescue robotics research and related technologies the world over. Hopping from continent to continent each year, this stop in North America continued the three-year rotation of North America - Europe - Asia.

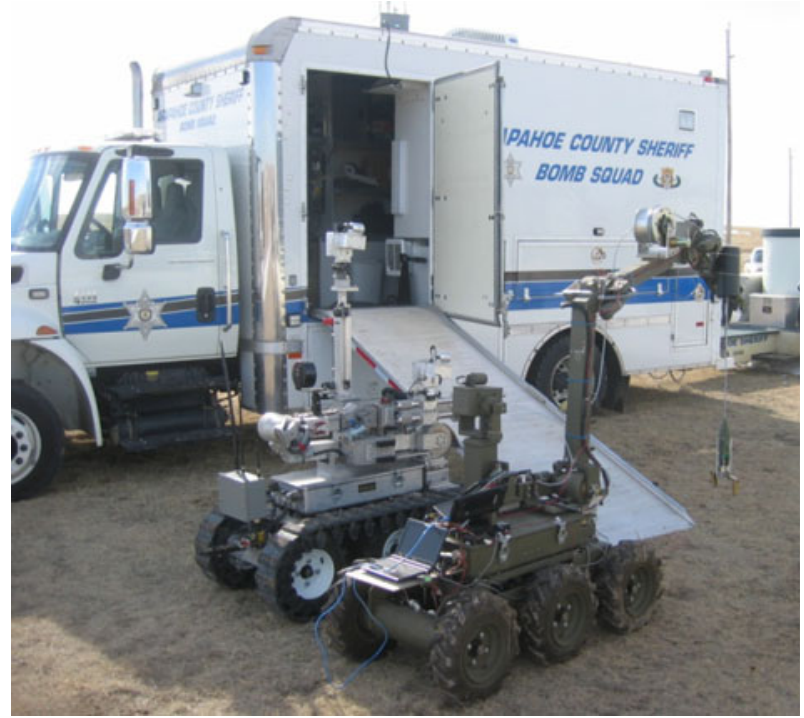

R. Voyles $(\bowtie)$

Department of Electrical and Computer Engineering, University of Denver, Denver, CO 80208-1500, USA

e-mail: rvoyles@du.edu

\section{J. A. Adams}

Electrical Engineering and Computer Science Department, Vanderbilt University, Nashville, TN 37235-1824, USA

e-mail: julie.a.adams@vanderbilt.edu 
As in years past, the meeting in Denver brought together researchers and practitioners for technical talks and demonstrations. This year's demonstrations focused on extending human capabilities through collaborations of people-animalsrobots (PAR). The first of two live demonstrations involved the Arapahoe County Bomb Squad and the first-ever public sector test of a "disruptor" breaching a car with gasoline in the tank. Disruptors are shaped water charges that "disassemble" suspected explosives energetically, before they have a chance to explode. This demonstration simulated a car bomb with explosives packed in the trunk. The disruptor was remotely placed directly under the gas tank of the car by the Arapahoe County Bomb Squad's Remotec Wolverine teleoperated robot. The Wolverine then backed away and the disruptor was detonated while an array of pressure sensors and high speed video cameras monitored the scene.

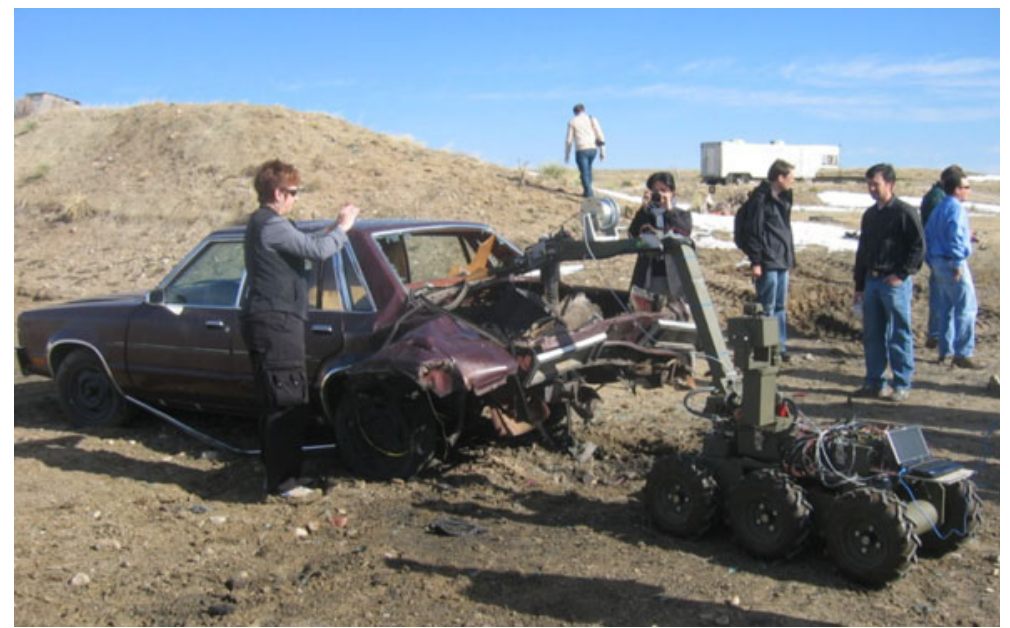

After the successful disruption of the simulated car bomb, a second Remotec Wolverine, deployed as a joint project between Lockheed Martin and the University of Denver Collaborative Mechatronics Lab, approached the vehicle for inspection. This second Wolverine was equipped with a marsupially-deployed CRAWLER robot (a.k.a. TerminatorBot) capable of crawling into the shattered vehicle to inspect structure and residue from the inside.

The second live demonstration paired the Front Range Rescue Dogs with the University of Denver Unmanned Systems Lab for a wilderness search and rescue task. The Front Range Rescue Dogs is an organization of search dogs and handlers trained to assist in missing person tracking in the mountainous region of the foothills and front mountain range of the Denver area. A wayward graduate student disappeared into the vastness of the University of Denver East Test Range. Two trained dogs from the Front Range Rescue Dogs tracked the missing person with their human handler. One of the dogs had a GPS beacon and transponder on its collar and a team of one UAV and two UGVs helped the human track the dogs' location. The UAV is able to keep pace with the dogs from above, relaying the dogs' location to the UGVs and human handler. The UGVs tracked the dogs' waypoints relayed by the UAV, homing in on the missing person at a slower pace. 


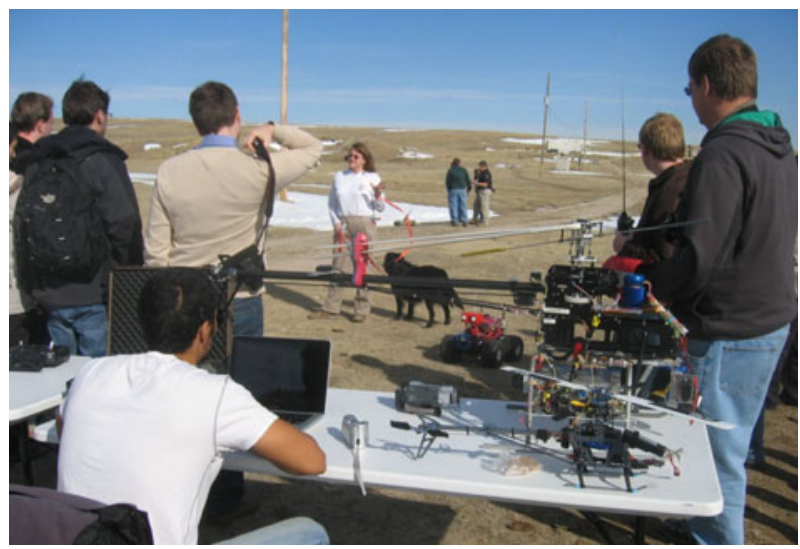

The technical sessions during SSRR spanned the gamut of applications and field trials including land, air, and water-based search and inspection. Technical topics included mechanism design, sensor integration, SLAM, search strategies, communication strategies, human/robot interaction, and victim stabilization. The papers presented in this special issue represent expansions of some of the best papers covering these topics.

The first paper by Yim and Laucharoen is a ground-breaking paper on robotic victim manipulation during rescue tasks. This novel research is likely the first of its kind on human patient manipulation and has many applications outside of search and rescue. The paper combines experimental studies of the forces required to manipulate a range of human patients in various ways with clever robotic "grippers" to effect these manipulations.

The paper by Birk et al. is a field report of unmanned aerial vehicle (UAV) trials at the European Land Robot Trials in Oulu, Finland and the Response Robot Evaluation Exercise in Disaster City, Texas, USA. A quadrotor UAV with GPSbased waypoint finding was used to host a camera for image mosaicking. The mosaicking approach is then integrated with SLAM results to enhance the photo maps.

Craighead examines the problem of robot operator performance in urban search and rescue applications. He applies a measure of the fractal dimension of the path followed by a robot to evaluate efficacy of a user's control over the robot and, thus, the efficacy of the human/robot interface. A virtual training environment based on the SARGE multi-player robot simulation game is used to test teleoperator control of a simulated Inuktun Extreme robot. Test results show the fractal dimension of the path provides a measure of quality of the operator's search strategy as well as ability to maintain situational awareness that is different from simple time-tocomplete metrics.

Elmogy et al. address the problem of optimal task allocation in a surveillance application with mobile robots. They use somewhat of an "international" marketbased approach in which the currencies and exchange rates are the priorities of the surveillance tasks and the efficacy of sensing agents.

Kleiner and Dornhege examine the problem of generating accurate maps for first responders in real-time. Without any dependence on dead reckoning data, which can be highly questionable in the chaotic environments of urban search and rescue, they 
present a two-step scan matching algorithm that uses only laser range finder and IMU data. They augment the resulting map with a user-guided global consistency check based on the outside perimeter of the building obtained from aerial images or ground-based images of the building's exterior walls. This self-contained approach is transportable to various robotic platforms and produces accurate maps on-the-fly.

Murphy et al. present a field report of unmanned marine vehicles deployed for bridge inspection after hurricane Ike hit land in Texas, USA. One surface vehicle, one tethered underwater vehicle and one autonomous underwater vehicle were tested with their sensor suites to examine debris fields around bridges and to detect "scour". The possibility of other littoral operations is also discussed.

Finally, in a paper that appears in a different issue of the journal, Scerri et al. examine the difficult and practical task of building entry during search and rescue. Multi-robot teams have been proposed to speed up search tasks through parallel operation, but search can't begin until a robot has entered the building of interest. Multi-robot teams present a unique challenge because entry points to the region of interest provide a "choke point" when multiple robots attempt access at the same time. Scerri et al. use simulation to evaluate a variety of motion heuristics to maximize throughput at the choke point.

We hope you enjoy this special issue and check the proceedings of IEEE SSRR for the many interesting articles that could not be accommodated in this journal. The SSRR proceedings are available on IEEExpert. 